
SciDoc

International Journal of Diabetology \& Vascular Disease Research (IJDVR)

ISSN:2328-353X

\title{
Analysis of Clinical Outcome and Healthcare Resource use in Insulin Treated Diabetic Patients Based on Self-Monitoring of Blood Glucose Levels
}

Research Article

Saragoni $\mathrm{S}^{1}$, Perrone $\mathrm{V}^{1}$, Buda $\mathrm{S}^{1}$, Genovese $\mathrm{S}^{2}$, Laurenzi $\mathrm{A}^{3}$, Mannucci $\mathrm{E}^{4}$, Degli Esposti $\mathrm{L}^{*}$

${ }^{1}$ CliCon S.r.l. Health, Economics and Outcomes Research, Ravenna, Italy.

${ }^{2}$ Department of Cardiovascular and Metabolic Diseases, IRCCS Multimedica, Sesto San Giovanni, Milan, Italy.

${ }^{3}$ Diabetes and Endocrinology Unit, Department of Internal Medicine, San Raffaele Scientific Institute, Milan, Italy.

${ }^{4}$ Diabetology, Careggi Hospital, Florence, Italy.

\section{Abstract}

Purpose: The purpose of the study hereof is to evaluate the appropriate use of blood glucose self-monitoring devices (strips), the risks of hypoglycemia, the incidence of hospitalization or death related to diabetes, and to calculate the use of healthcare resources based on self-monitoring of blood glucose (SMBG), in insulin treated patients.

Methods: A retrospective study was conducted based on current administrative flows. The study included patients having at least one insulin prescription from November 1, 2009 to April 30, 2011 (inclusion period). The index date was defined as the date within the inclusion period in which the patient presented the first insulin prescription. All patients were under observation for 18 months starting from the index date (observation period). Screening was conducted during the 12 months prior to the observation. Patients who resorted to self-monitoring of blood glucose were identified by the number of test strips prescribed by the National Health Service. Consumption data refer to the observation period of the analysis. Results: 3,890 patients were included, $26.4 \%$ of which resorted to self-monitoring less than once a day whereas $10 \%$ never did. The incidence of hypoglycemia was linked to daily use or lack of use of SMBG (27.3 vs. 10.6). Based exclusively on the cost of hospitalization, lack of use of SMBG resulted in an increased consumption of healthcare resources.

Conclusions: Based on the results presented, we hope that this study will encourage development and implementation of educational and training programs on self-management of diabetes, and of models on adopting clinical decisions and on healthcare provided, based on monitored values.

\section{Introduction}

Diabetes is a chronic disease caused by elevated blood glucose levels due to altered insulin levels or function. The disease has an important hereditary component and can be classified as Type 1 (in approximately $10 \%$ of cases) or Type 2 (in $90 \%$ of cases) [1].

According to the International Diabetes Federation estimates, over 300 million people in the world are affected by diabetes [2]. The last ISTAT report, from the year 2012, shows that diabetes has a prevalence of $5.5 \%$ in Italy. Prevalence rises to $15 \%$ in age groups between 65 to 74 years of age, and one out of five people are affected after the age of $75(20.3 \%$ prevalence) [3]. The percent of affected patients in Italy has risen from the year 2000 to the year 2012. Prevalence has risen from $3.7 \%$ to $5.5 \%$ (ISTAT data) and diabetes will continue to diffuse during the years as the population progressively ages and the risk factors increase [3].

Diabetes alone, but mainly the acute and chronic complications related to it, play an important role on the social and financial impact of this metabolic disorder. Micro-vascular and macrovascular complications, other than being extremely impairing and compromising the function of the main organs, have a major social and financial impact on several national systems.

The huge social and financial burden of this disease strongly affects the National Healthcare's budget. Financial international studies estimate that at least $6-15 \%$ of the healthcare cost in

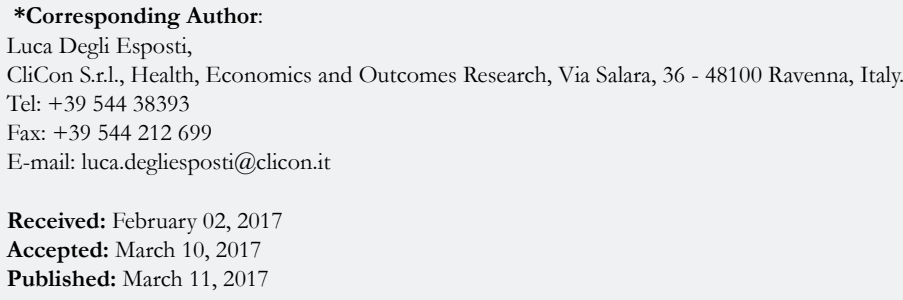

Citation: Saragoni S, Perrone V, Buda S, Genovese S, Degli Esposti L, et al., (2017) Analysis of Clinical Outcome and Healthcare Resource use in Insulin Treated Diabetic Patients Based on Self-Monitoring of Blood Glucose Levels. Int J Diabetol Vasc Dis Res,. 5(2), 183-188. doi: http://dx.doi.org/10.19070/2328-353X-1700038 
Europe and the United States is used by diabetic patients. A recent analysis, conducted by the ARNO Diabetes Observatory, shows that the average annual cost for managing diabetes by the Italian National Health Service (NHS) amounts to $€ 3,899$; the majority of costs are due to hospitalization (over $70 \%$ ), 15\% are due to related complications and comorbidity, $4 \%$ are due to prescription medications and the rest are due to lab work, diagnostic exams and doctor's visits [4].

Even though the studies conducted on the cost of diabetes adopted different methods and were not always comparable, they all agreed in asserting that this cost is inevitably destined to increase due to the increased prevalence of the disease and also due to the increased healthcare cost for treating the disease and its complications [5-7].

Current scientific evidence shows that compliance to therapy and self-monitoring of blood glucose levels are crucial in managing the disease over time, having the ultimate goal of stabilizing blood glucose levels and reducing the progression and onset of serious cardiovascular complications and correlated costs [8].

The guidelines stated by the Italian scientific societies AMD (Associazione Medici Diabetologi) and SID (Società Italiana di Diabetologia), emphasize the importance of appropriate use of self-monitoring of blood glucose levels based on the type of diabetes and on the patient's clinical profile.

The purpose of the study hereof is to evaluate the appropriate use of blood glucose self-monitoring devices (strips), the risks of hypoglycemia, the incidence of hospitalization or death related to diabetes, and to calculate the use of healthcare resources based on $\mathrm{SMBG}$, in insulin treated patients.

\section{Methods}

\section{Data Resources}

Data of analyzed patients was obtained from samples in administrative databases of Local Healthcare Units (LHU) in Tuscany and Emilia Romagna having a patient population of roughly one million individuals, representative of the demographic structure of the Italian population.

The following archives were analyzed: the health-assisted subjects' database, containing the demographic characteristics of the analyzed subjects (gender and age); medications prescription databases, containing all the information regarding the drug treatments provided to the analyzed subjects and reimbursed by the NHS, such as for example the Anatomical-TherapeuticChemical (ATC) code of the prescribed drug, the number of packages, the number of units per package, the dose, the cost per unit and the prescription date; Hospital discharge database, containing information regarding hospitalization, such as admission and discharge date, main and additional diagnosis, encoded based on the International Classification of Diseases, IX Revision, Clinical Modification (ICD-9-CM); Ambulatory Care Specialist, containing a record of specialized services (doctor visits, lab exams, diagnostic exams), performed on analyzed subjects affiliated with the NHS, also including codes ICD-9-CM related to the exemptions by disease; Medical devices, containing information regarding the use of medical devices on behalf of facilities affiliated with the NHS; the clinical laboratory databases (will provide information on disease management based on the evaluation of related lab parameters).

In accordance with the Privacy regulation, the patient's identification code was encrypted and all personnel responsible for processing data was not provided with any information which could be directly or indirectly used in identifying the patient. The anonymous patient identification contained in each archive allowed the different databases to be linked. This integrated database was used to build a population data bank containing the individual chronological and analytical profile of the entire population pertaining to the participating LHU.

In accordance with the regulation in effect on conducting an observational study [10], the study hereof has been notified to the Local Ethical Committee of every participating LHU.

\section{Patients Included}

A cohort retrospective analysis was conducted. The study included patients having at least one insulin prescription (ATC code $=\mathrm{A} 10 \mathrm{~A}$ ) from November 1, 2009 to April 30, 201 (inclusion period). The date within the inclusion period in which the patient presented the first insulin prescription was defined as the (index date). All patients were under observation for 18 months starting from the index date (observation period). Screening was conducted during the 12 months prior to the observation.

During the descriptive analysis, all patients were divided into three cohorts based on the treatment received during the observation period: Basal insulin (ATC codes: A10AC01, A10AC04, A10AE04, A10AE05); Basal + Mealtime insulin (ATC codes: A10AC01, A10AC04, A10AE04, A10AE05, A10AB01, A10AB04, A10AB05, A10AB06, A10AD01, A10AD04, A10AD05); Mealtime insulin (ATC codes: A10AB01, A10AB04, A10AB05, A10AB06).

Patients who transferred to another LHU or who died during the observation period were not included in the analysis.

\section{Definition and Outcome of the Study}

During the observation period we evaluated whether patients resorted to self-monitoring of blood glucose levels and obtained information on all assistance provided and recorded all blood glucose measurements on an empty stomach.

Whether or not patients resorted to self-monitoring of blood glucose was evaluated through the flow of medical devices and identified by the number of strips prescribed, necessary for the blood glucose self-monitoring devices, provided by the NHS.

During the observation period, plasma glucose levels were identified based on glycemic levels on an empty stomach. Concentrations $<70 \mathrm{mg} / \mathrm{dl}$ indicated a state of hypoglycemia; concentrations ranging from $70-99 \mathrm{mg} / \mathrm{dl}$ indicated normal glucose levels; concentrations ranging from 100-125 mg/dl indicated altered glucose levels and concentrations $\geq 126 \mathrm{mg} /$ $\mathrm{dl}$ indicated diabetes (which was confirmed by taking a second measurement). 
During the observation period, the following causes for diabetes-related hospitalization were identified: hypoglycemia [hypoglycemic coma-iatrogenic hyperinsulinism-nondiabetic insulin coma (codes ICD-9-CM: 251.0), other causes of hyperinsulinism (ICD-9-CM code: 251.1), non-specified hypoglycemia (ICD-9-CM code: 251.2)]; cardiovascular diseases [heart attack and other ischemic heart diseases (codes ICD-9CM: 410-414), stroke and other types of brain damage (ICD-9CM codes: 430-438), arrhythmias (ICD-9-CM codes: 427), heart failure (ICD-9-CM code: 428), atherosclerosis and aneurisms (ICD-9-CM codes: 440-442), other cardiovascular causes (ICD9-CM codes: 401-405)]; kidney disease [kidney failure (ICD-9CM codes: 584, 585)]; other diagnosis related to diabetes [ other diseases of retina (ICD-9-CM code: 362), diabetes mellitus (ICD9-CM code: 250), arthrosis (ICD-9-CM code: 715), fractures of the neck of the femur (ICD-9-CM codes: 820), fractures of the tibia and peroneal bone (ICD-9-CM codes: 823)].

\section{Cost Analysis}

The cost analysis was conducted from the perspective of the Italian NHS. The costs were obtained from the analyzed archives and only the costs directly related to the disease studied were taken into consideration. Consumption data refer to the observation period of the analysis. For treatments, we considered the drug's tag price at the time of purchase; outpatient services were valued based on a regional rate table; the cost of the self-monitoring strips, for home based monitoring, was calculated based on the purchase price by healthcare facilities (based on the price CON. S.I.P. - Concessionaria Servizi Informativi Pubblici-Public Service Information Authority) and finally, hospitalization price of a single hospital stay derived from the DRG (Diagnosis related Group) assigned regionally, which eventually increased in case the hospital stay was prolonged over the threshold value of each DRG.

\section{Statistical Analysis}

Continuous variables are reported as average \pm standard deviation (median and range where necessary); categorical variables are reported as absolute numbers or percentages. P-values below 0.05 are considered statistically significant. All data analysis was conducted using SPSS-Windows 18.0.

\section{Results}

A total of 3,890 patients, $52 \%$ of which were males (average age ( \pm SD) $66.2 \pm 15.1$ years), was included in the study (Table 1 ). During the observation period, $68.3 \%$ of patients were under Basal + Mealtime insulin treatment, whereas $15.7 \%$ were under Basal insulin treatment and 16\% were under Mealtime insulin treatment (Table 1).

Only $26.4 \% \quad(n=1,026)$ of all patients analyzed resorted to SMBG less than once a day during the observation period; $28.8 \%(n=1,120)$ of the patients resorted to $1-2$ SMBG per day and $34.7 \%(n=1,350)$ resorted to more than 2 SMBG per day. Only $10 \%(n=394)$ of all patients analyzed never resorted to daily SMBG levels (Figure 1).

Table 2 shows the demographic characteristics of the patients included in the study, stratified based on frequency of blood glucose self-monitoring.

The incidence of hypoglycemia in insulin treated diabetic patients was linked to the daily lack of use or use of SMBG (27.3 vs. 10.6) (Figure 2), having twice the hospitalization rate (60.8 vs 30$)$ (Figure 3).

Similarly to the incidence of hypoglycemia and hospitalization, based exclusively on the cost of hospitalization, the lack of use of SMBG resulted in an increased consumption of healthcare resources during the observation period ( $€ 2,500$ vs $€ 1,500$ ) which exceed 66\% (Figure 4).

\section{Discussion}

Even though home based SMBG levels in diabetic patients is

Table 1. Characteristics of Patients Included in the Study based on the Insulin Treatment Received during the Observation Period.

\begin{tabular}{|c|c|c|c|c|}
\hline \multirow{2}{*}{ Patients } & \multirow{2}{*}{ Total } & \multicolumn{3}{|c|}{ Type of Insulin Treatment } \\
\cline { 3 - 5 } & & Basal & Basal + Mealtime & Mealtime \\
\hline $\mathrm{n}(\%)$ & $3,890(100.0)$ & $610(15.7)$ & $2,657(68.3)$ & $623(16.0)$ \\
\hline Age (average $\pm \mathrm{SD})$ & $66.5 \pm 15.1$ & $67.4 \pm 12.7$ & $65.5 \pm 15.2$ & $69.7 \pm 16.0$ \\
\hline Males $(\%)$ & 52.2 & 54.4 & 52.5 & 48.8 \\
\hline
\end{tabular}

Figure 1. Frequency of Self-Monitoring of Blood Glucose Levels in Insulin Treated Diabetic Patients.

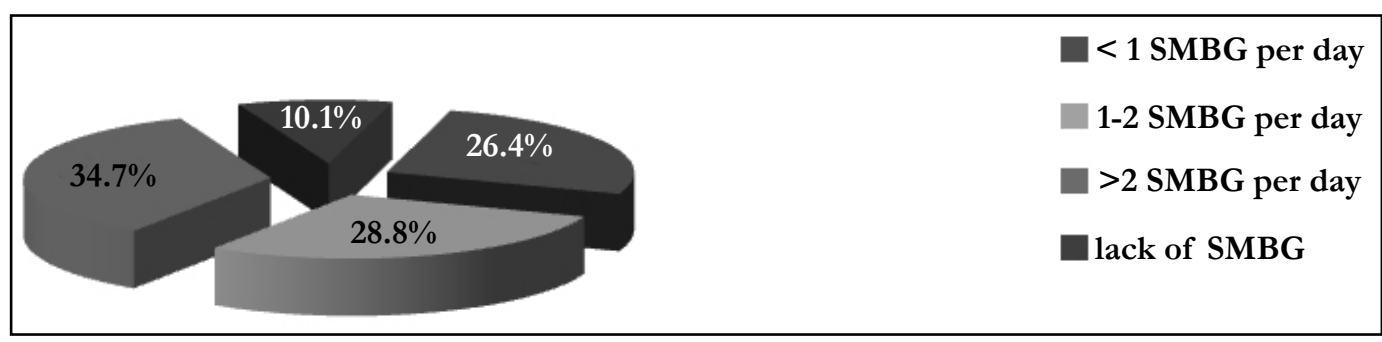

Abbreviation: self-monitoring of blood glucose, SMBG; 
Table 2. Demographic Characteristics of Patients Included in the Study, Stratified based on Frequency of Self-Monitoring of Blood Glucose Levels.

Frequency of SMBG levels

\begin{tabular}{|c|c|c|c|c|c|c|c|c|}
\hline Patients & $\begin{array}{c}<\mathbf{1} \\
\text { SMBG per } \\
\text { week }\end{array}$ & $\begin{array}{c}\mathbf{1 - 2} \\
\text { SMBG per } \\
\text { week }\end{array}$ & $\begin{array}{c}\text { SMBG per } \\
\text { week }\end{array}$ & $\begin{array}{c}\mathbf{1 - 2} \\
\text { SMBG per } \\
\text { day }\end{array}$ & $\begin{array}{c}\text { 3-4 } \\
\text { SMBG per } \\
\text { day }\end{array}$ & $\begin{array}{c}\text { SMBG } \\
\text { SMer } \\
\text { day }\end{array}$ & $\begin{array}{c}\text { Lack of } \\
\text { SMBG }\end{array}$ & $\begin{array}{c}\text { P value } \\
\text { n }(\%)\end{array}$ \\
\hline $84(2.2)$ & $328(8.4)$ & $614(15.8)$ & $1,120(28.8)$ & 1,127 & 29.0 & $223(5.7)$ & $394(10.1)$ & \\
\hline Average age & $65.7 \pm 13.9$ & $66.8 \pm 15.2$ & $67.9 \pm 13.5$ & $67.7 \pm 13.5$ & $66.6 \pm 14.1$ & $47.9 \pm 17.2$ & $70.7 \pm 15.9$ & $<0.001$ \\
\hline Males $\%$ & 59.5 & 57.0 & 54.1 & 52.1 & 50.7 & 45.7 & 52.0 & 0.104 \\
\hline
\end{tabular}

Abbreviation: self-monitoring of blood glucose, SMBG;

Figure 2. Incidence of Hypoglycemia in Insulin Treated Diabetic Patients Based on Lack of use or use of Self-Monitoring of Blood Glucose Levels.

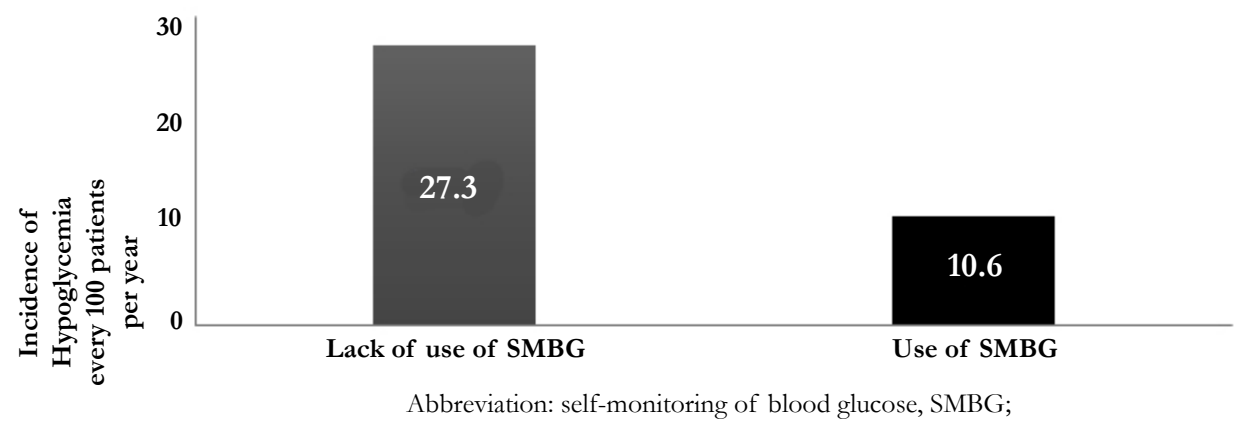

Figure 3. Incidence of Hospitalization or Death in Insulin Treated Diabetic Patients Based on Lack of Use or Use of SelfMonitoring of Blood Glucose levels.

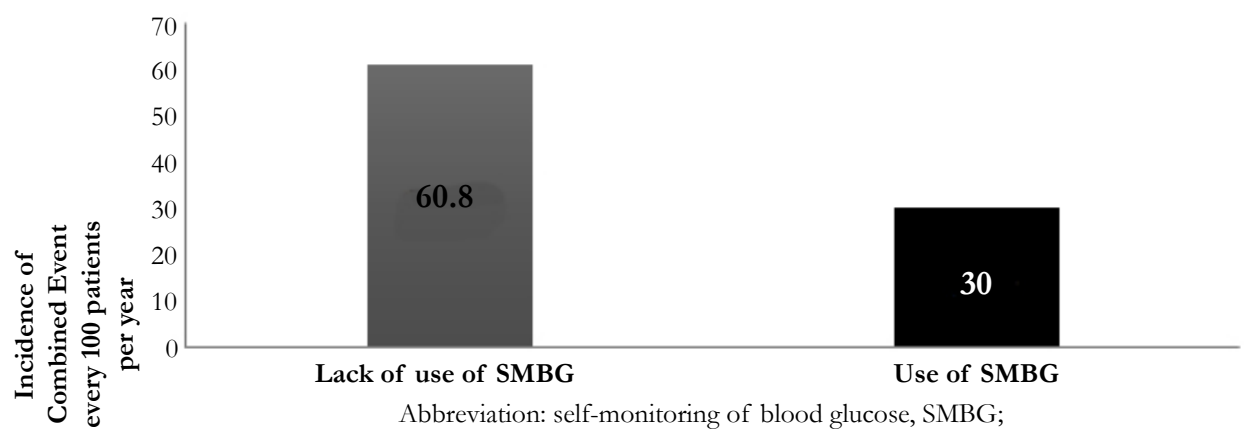

Figure 4. Consumption of Healthcare Resources in Insulin Treated Diabetic Patients Based on Lack of Use or Use of SelfMonitoring of Blood Glucose Levels During the Observation Period.

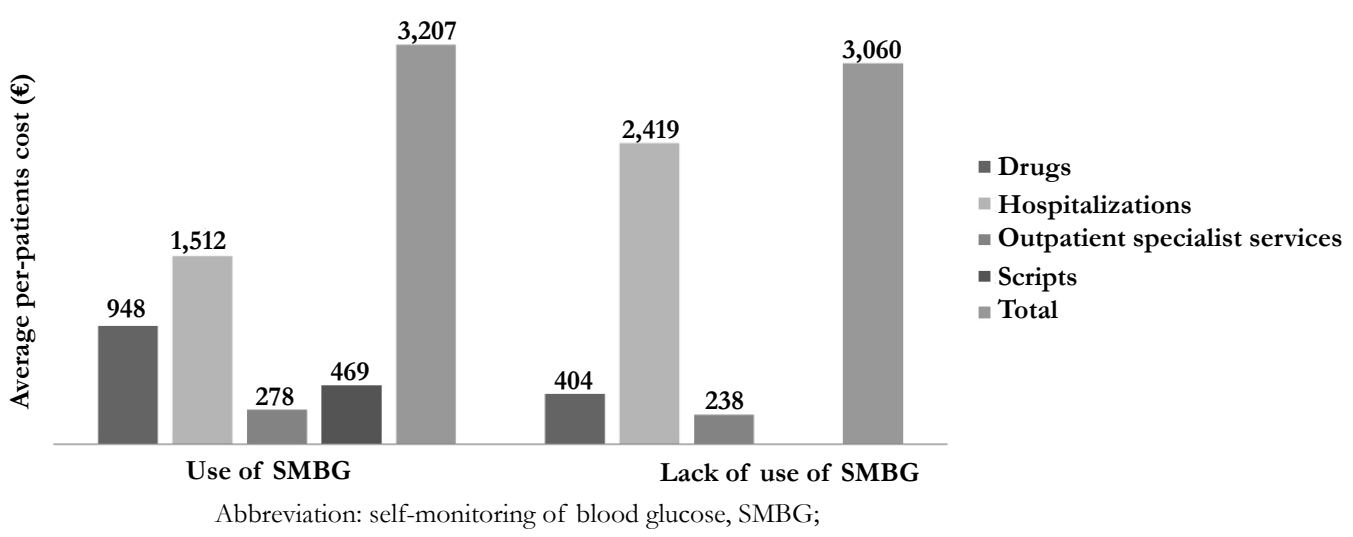


highly recommended [11], our study shows that only one third of the population analyzed regularly used the blood glucose selfmonitoring methods based on the guidelines available today [12]. In $65 \%$ of the insulin treated patients, the self-monitoring strips were used less than once a day. In $55 \%$ of the insulin treated patients, the self-monitoring strips were used less than twice a day. Ten percent of patients did not use the strips at all.

Self-monitoring relies on healthcare devices, blood glucose meters and strips, but it is prescribed by a doctor and it is self-administered by the patient based on a well-defined plan, therefore, like with any other treatment, problems may arise due to compliance and motivation. The term SMBG levels is therefore a true treatment that needs to be prescribed based on specific directions and procedures. The family doctor or endocrinologist should select the most suitable approach in order to easily evaluate how the set therapeutic goals are being met, in order to modify the therapy based on the blood glucose values and in order to prevent micro/ macro-vascular complications related to diabetes [11].

Several clinical guidelines based on evidence, recommend that the frequency of SMBG should be determined based on the individual patient's characteristics, on the type of diabetes and on the therapeutic strategy adopted $[11,12]$.

The results of our study are consistent with the results of previously conducted studies which evaluated the use of SMBG in different countries in Europe [12]. European studies on the use of strips, published based on the guidelines set by the Diabetologists Doctor Association, in collaboration with the Italian Diabetologist Society [12], show that the use of strips per-capita in Italy is lower by $25-29 \%$ compared to the average European use; current evidence may indicate an underuse of SMBG in Italy, resulting in potentially negative effects on the diabetic patient's health and on general healthcare costs.

Several studies have shown that the appropriate use of SMBG is crucial to successfully managing diabetes, not only providing both patients and doctors with the necessary information to optimize therapy but also providing them with a tool to detect the potential hypoglycemia and to reduce the risk of post-prandial hyperglycemia [13-15].

Regarding the financial impact of diabetes in Italy, a recent analysis by the Ministero della Salute (Health Department), estimates that the annual total cost of this disease is of 5.17 million euros, which is equal to $6.65 \%$ of the overall national healthcare cost. The cost of providing healthcare assistance to a diabetic patient increases by 3 to 4 times in case of cardio/cerebrovascular complications alone or micro-vascular complications alone (affecting kidneys, retina and peripheral nervous system) and by 5 times in case of both types of complications together [16].

The results presented by this study are supported by current scientific evidence, proving how the correct and constant use of SMBG is the most effective way to reduce the onset of vascular complications affecting target organs and to reduce the annual hospitalization incidence $[13,17]$.

The financial evaluations based on evidence are an integrated part of the optimized use of healthcare resources and of the recommended specific strategies for managing the disease. A recent analysis conducted in Italy has shown how a diabetic patient who is closely being followed and who is correctly complying with the prescribed treatment, roughly costs $€ 1,800$ per year, while a patient having serious complications can cost over $€ 10,000$, in addition to the social obligations deriving from the possibility that the complications progress [16].

Based on this, and aside from the geographic variety and variability of the results obtained in the randomized clinical trials and observational studies, the use of SMBG could be useful in a global educational program which will help patients and healthcare providers maximize the efficacy of treatment and optimize behavior based on the results of the blood glucose levels [18-22].

Furthermore, we must underline that when interpreting the data regarding healthcare consumption presented by this study, a few limitations should be taken into account. It was not possible to assign each patient with a specific indicator of disease severity given that this information was not found in the administrative database. For this reason the patient selection in the study could be biased. With this in mind, we must underline that the frequency of self-monitoring also varies based on the patient's clinical state. As a matter of fact, resorting to self-monitoring is lower in patients having a more severe clinical condition (i.e. patients affected by dementia, or by progressive strokes which resulted in severe physical impairment), and in these cases lack of use of SMBG could be a marker of severity of the disease.

Finally, unhealthy life-styles and inadequately complying with the prescribed drug treatment, are associated with a lower frequency of SMBG and a higher risk of complications. In these cases lack of use of SMBG could be a marker of unhealthy behavior and lack of discipline.

As a final note, since the results of this study refer to a small sample of Italian LHUs, they are not comprehensive results but require further investigations on larger patient populations.

\section{Conclusion}

There is current solid evidence in literature regarding the crucial role played by the interaction between diabetic patients and healthcare professionals in meeting the set therapeutic goals. For this reason, SMBG levels should rise from a shared decision between patients and healthcare professionals. Based on the results presented, we hope that the study hereof will encourage development and implementation of educational and training programs on self-management of diabetes, and of models on adopting clinical decisions and on healthcare provided, based on monitored values.

\section{References}

[1]. World Health Organization. Diabetes. http://www.who.int/diabetes/en/.

[2]. International Diabetes Federation. http://www.idf.org.

[3]. ISTAT. Il diabete in Italia. http://www.istat.it/it/archivio/71090.

[4]. Cineca Dipartimento SISS Sanità (2011) Osservatorio ARNO Diabete. Il profilo assistenziale della popolazione con diabete. XVII - Collana "Rapporti ARNO".

[5]. Trogdon JG, Hylands T (2008) Nationally Representative Medical Costs of Diabetes by Time Since Diagnosis. Diabetes Care. 31(12): 2307-2311.

[6]. Kim TH, Chun KH, Kim JH, Han SJ, Kwak J, et al., (2012) Direct Medi- 
cal Costs for Patients with Type 2 Diabetes and Related Complications: A Prospective Cohort Study Based on the Korean National Diabetes Program. J Korean Med Sci. 27(8): 876-82.

[7]. Gnavi R, Karaghiosoff L, Dalmasso M, Bruno G (2006) Validazione dell'archivio regionale di esenzione per diabete della Regione Piemonte: vantaggi e limiti per un uso epidemiologico. Epidemiol Prev. 30(1): 59-64.

[8]. Operatori Sanitari di Diabetologia Italiani (OSDI). Raccomandazioni di trattamento assistenziale in campo diabetologico. Le position statement OSDI 2011/2012.

[9]. AIFA Guideline for the classification and conduction of the observational studies on medicines.

[10]. Associazione Medici Diabetologi (2012) Raccomandazioni per l'autocontrollo della glicemia nel paziente diabetico. Revisione. 1-8.

[11]. Associazione Medici Diabetologi (AMD), Società Italiana di Diabetologia (SID) - Standard italiani per la cura del diabete mellito 2014

[12]. Giaccari A, Grassi G, Ozzello A (2012) Self-Monitoring of Blood Glucose: Guideline Application Rather than Utilization Restrictions on Testing Strips Has Potential to Reduce Diabetes Healthcare Costs in Italy. Diabetes Technol Ther. 14(10): 862-7.

[13]. Franciosi M, Pellegrini F, De Berardis G, Di Nardo B, Kaplan SH, et al., (2001) The impact of blood glucose self-monitoring on metabolic control and quality of life in type 2 diabetic patients: an urgent need for better educational strategies. Diabetes Care. 24(11): 1870-1877.

[14]. Giorda C, Nada E, Chiò E (2007) Analisi della prescrizione di stri- sce per l'automonitoraggio glicemico nella Regione Piemonte in relazione ad alcune variabili cliniche e assistenziali. Abstract congresso AMD, Sorrento.
[15]. Miikkulainen K, Caruso A, Mast O, Zhang R, Borisenko O (2016) Systematic literature review of use of blood glucose monitoring in phase III clinical studies of insulin analogs. BMC Endocr Disord. 16: 21.

[16]. CARE Costi dell'assistenza e risorse economiche. Anno 13 Maggio - Giugno 2011.

[17]. Karter AJ, Ackerson LM, Ferrara A, Liu L, Selby JV, et al., (2001) Self-monitoring of blood glucose levels and glycemic control: the Northern California Kaiser Permanente Diabetes registry. Am J Med. 111(1): 1-9.

[18]. Schwedes U, Siebolds M, Mertes G, SMBG Study Group (2002) Mealrelated structured self-monitoring of blood glucose: effect on diabetes control in non-insulin-treated type 2 diabetic patients. Diabetes Care. 25(11): 1928-1932.

[19]. Jansen JP (2006) Self-monitoring of glucose in type 2 diabetes mellitus: a Bayesian meta-analysis of direct and indirect comparisons. Curr Med Res Opin. 22(4): 671-681.

[20]. Clar C, Barnard K, Royle P, Waugh N, Cummins E, et al., (2010) Selfmonitoring of blood glucose in type 2 diabetes: systematic review. Health Technol Assess Winch Engl. 14(12): 1-140.

[21]. Karter AJ, Parker MM, Moffet HH, Chan J, Ettner SL, et al., (2006) Longitudinal study of new and prevalent use of self-monitoring of blood glucose. Diabetes Care. 29(8): 1757-1763.

[22]. Bosi E, Scavini M, Ceriello A, Tiengo A, Bonizzoni, et al., (2013) Intensive structured self-monitoring of blood glucose and glycemic control in noninsulin-treated type 2 diabetes: the PRISMA randomized trial. Diabetes Care. 36(10): 2887-2894. 